Fetal Diagnosis

and Therapy
Fetal Diagn Ther 2009;25:297-303

DOI: 10.1159/000235875
Received: June 5, 2009

Accepted: June 9, 2009

Published online: September 22, 2009

\title{
Should We Customize Fetal Growth Standards?
}

\author{
F. Figueras ${ }^{a, b} \quad$ J. Gardosic ${ }^{c}$ \\ a Maternal-Fetal Medicine Department, Hospital Clínic, Universitat de Barcelona, and berinatal Research Group, \\ Institut d'Investigacions Biomèdiques August Pi i Sunyer, Barcelona, Spain; 'West Midlands Perinatal Institute, \\ Birmingham, UK
}

\section{Key Words}

Intrauterine growth restriction • Small for gestational age • Birth weight

\begin{abstract}
Several maternal and fetal physiological characteristics account for a substantial proportion of the variation in birth weight. These characteristics can be used to calculate an individualized optimal birth weight and to adjust or 'customize' the birth weight standard. Customized birth weight standards improve the distinction between constitutional and pathological smallness, and there is evidence that this finding can be extrapolated into the fetal period to evaluate intrauterine growth, but further studies are required to evaluate and quantify the effectiveness of customized versus conventional charts in improving the diagnosis of pathological smallness before birth.

Copyright $\odot 2009$ S. Karger AG, Base
\end{abstract}

\section{Introduction}

Intrauterine growth restriction (IUGR) is defined as a failure of the fetus to reach its growth potential. It is a major contributor to perinatal mortality, being etiologically responsible for $50 \%$ of perinatal deaths occurring preterm and $20 \%$ at term [1]. This condition is also associated with intrapartum distress, metabolic acidosis, cerebral palsy
[2] and with a wide range of neurocognitive and neurobehavioral disruptions that persist into childhood [3]. Finally, there is growing evidence of the association between growth restriction and metabolic syndrome in adulthood [4]. However, the identification of IUGR remains elusive, since the growth potential could not be precisely quantified. Because most cases of growth restriction are also small for gestational age (SGA), birth weight or estimated fetal weigh is used as surrogate. Nevertheless, this could be misleading, since not all small babies are growth restricted and not all growth-restricted infants are small [5]. Thus, the differentiation between IUGR and SGA represents a major challenge in modern obstetrics.

Most instances of true growth restriction correspond with cases of placental insufficiency [6]. Thus, intrauterine placental function evaluation by umbilical artery Doppler is the clinical standard to distinguish between SGA and IUGR [7-9], and there is evidence that umbilical Doppler ultrasound use in these pregnancies improves many obstetric care outcomes and reduces perinatal deaths [10]. While abnormal umbilical Doppler is associated with adverse perinatal and neurodevelopmental outcome [11-14], small fetuses with normal umbilical artery Doppler are considered to represent one end of the normal size spectrum, and the importance of managing them completely differently from true IUGR babies has been stressed $[15,16]$. However, growing evidence suggests that a considerable proportion of fetuses with the diagnosis of SGA have true growth restriction in spite of a normal um-

\section{KARGER \\ Fax +41613061234 E-Mail karger@karger.ch} www.karger.com

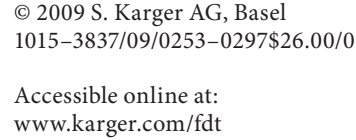

Francesc Figueras

Maternal-Fetal Medicine Department

Hospital Clinic

ES-08028 Barcelona (Spain)

Tel. +34932 275 600, Fax +34932 275 605, E-Mail ffiguera@clinic.ub.es 
bilical artery Doppler $[11,12,17]$. This contention seems to be more relevant near term, when most instances of adverse outcome attributable to growth restriction occur in babies with normal umbilical Doppler [18].

The adjustment of the expected birth weight by its determinants has been called 'customization', and it has been proposed to improve the distinction between SGA and IUGR. This paper aims to review the evidence for incorporating customization into clinical practice as a primary tool to better define IUGR both prenatally and in the neonatal period.

\section{Physiological Factors Influencing Birth Weight}

Gestational age is a major determinant of fetal growth, with an almost linear effect throughout the third trimester resulting in an average weight gain of about $25 \mathrm{~g}$ per day $[19,20]$. When pregnancies are not dated by ultrasound, growth modeling results in an artifactual flattening beyond 40 weeks, because the proportion of misdated pregnancies in this period is substantial. Apart from the length of gestation, fetal growth is significantly influenced by many other fetal and maternal physiological factors such as sex, parity, maternal height and weight and ethnicity. The effect of fetal gender on birth weight differs across studies (232-310 g at 280 days) [21-28]. Most of this variability is secondary to absolute differences in the average birth weight of the population on which each study was carried out. The effect of maternal weight at booking has also been found positively associated with birth weight [21-28], with a negative effect at both extremes (underweight and obesity) [26]. Similarly, maternal height also has a positive correlation with a plateau effect at the upper extreme. On average, women with a previous delivery have babies $87-110 \mathrm{~g}$ heavier [21-28], but this effect decreases at higher parities, and some series have even demonstrated a negative effect in grand multiparae women [22]. Ethnicity has also an important effect on fetal growth [29] and birth weight [21-26, 28, 30]. Graafmans et al. [30] compared birth weights in 7 Western European countries and found substantial differences between countries. They concluded that to improve the identification of growth-restricted infants, population-specific standards for birth weight should be developed. However, deriving standards based on geography or ethnicity alone would neglect the effect on fetal growth of other maternal and fetal characteristics secondarily associated with ethnicity. Not only ethnicity could be considered a measure of 'biological' difference, since other social and environmental variables greatly differ between different ethnicities.

Overall, gender, parity, maternal height, weight and ethnicity explain a $20-35 \%$ of the variability of birth weight at term [21-26]. In addition, other maternal physiological variables such as age [22], educational [31], socioeconomic [32] and marital status [33] or altitude of the area of residence [34] also have a significant albeit less relevant effect on birth weight. Similarly, paternal height has been demonstrated to affect birth weight [35], but this effect is relatively minor and the information is often not available.

\section{Pathological Factors Influencing Fetal Birth Weight}

Cigarette smoking during pregnancy is a strong dosedependent risk factor for SGA [36]. Smoking alone independently explains 9 and $12 \%$ of preterm and term cases of SGA [37] and has been highlighted as the single largest modifiable risk factor affecting the growth of unborn infants in developed countries [38]. Other major prenatal pathological conditions that have relevant effects on fetal growth are diabetes and hypertensive disorders [22, 39]. In addition, congenital malformations are frequently associated with impaired fetal growth [40]. Finally, infants born preterm have a higher incidence of SGA than infants born at term. This is not only because IUGR is a common indication for delivery before term. Indeed, spontaneous preterm delivery or premature rupture of membranes are consistently associated with SGA [27, 4143] and both conditions share many risk factors, such as obesity, smoking or black ethnicity [42]. It has been speculated that the initiation of parturition is a fetal adaptive response to placental insufficiency.

This latter aspect makes it important to construct standards on fetuses rather than on neonates. The need to base preterm weight standards on fetal rather than on neonatal weights is illustrated in figure 1 . Ultrasound-estimated fetal weights are plotted from 480 low-risk pregnancies having a routine third trimester ultrasound. Whereas population-based standards [44] only show $1.7 \%$ of the population as SGA (below the 10th centile), fetal standards [23] identified $9.7 \%$ as SGA.

\section{Methodological Aspects}

The adjustment of the expected birth weight by its physiological and pathological determinants has been called 'customization' since the original report of this 
Fig. 1. Plot of 480 estimations of fetal weight measured at routine third trimester ultrasound in a low-risk Spanish population, with 10 th and 90 th centile lines based on the local neonatal weight standard (dotted lines) and the fetal weight standard, adjusted to a Spanish population average (maternal height $161 \mathrm{~cm}$, weight $59 \mathrm{~kg}$ ). The graphs show that while only 8 cases (1.7\%) were found to be SGA by the neonatal weight standard, the fetal weight standard identified 45 cases $(9.4 \%)$ as SGA.

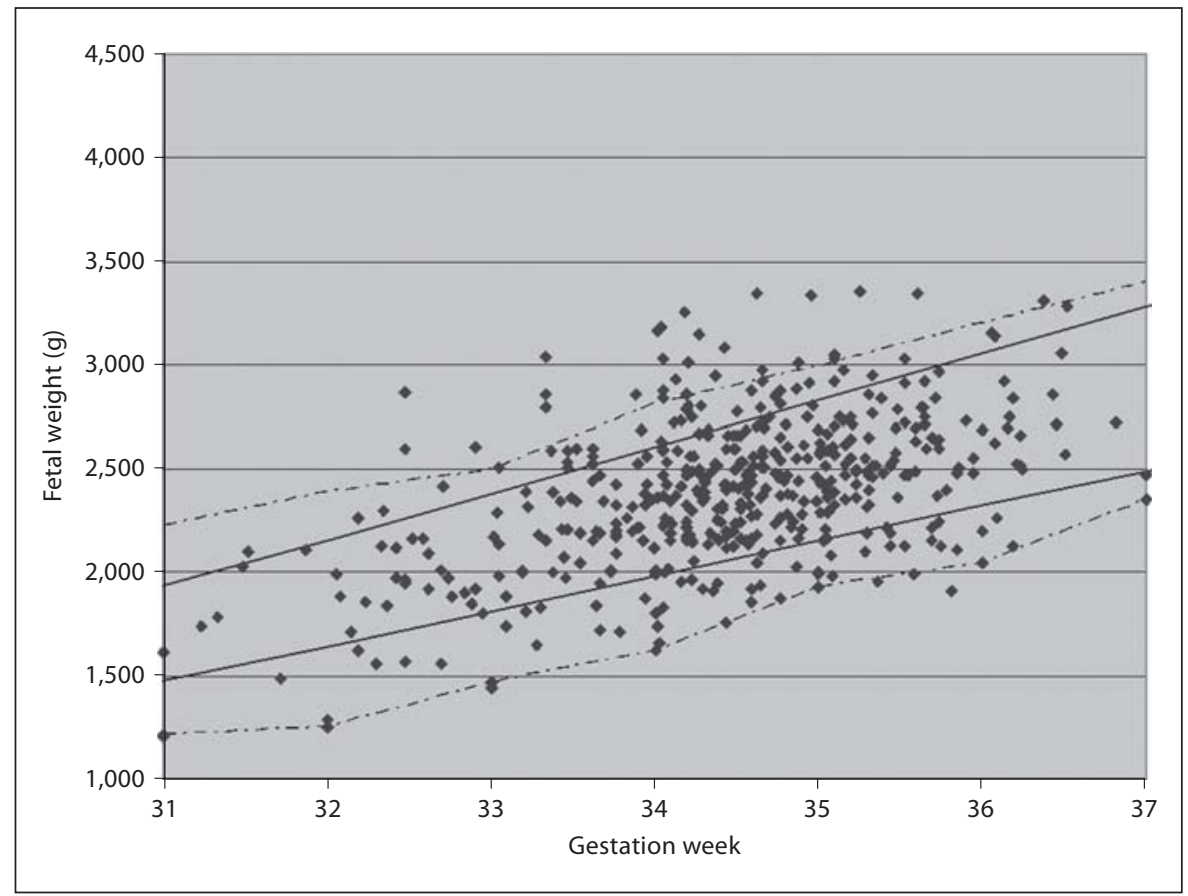

method [45]. Several standards for birth weight customization have been published, and some methodological aspects are noteworthy.

\section{Design}

Ultrasound dating is much more accurate than menstrual dating [46]. Because the distribution of menstrual dating error is positively skewed, and it is more frequent to have a long first menstrual phase than a short one, there is a systematic overestimation of gestational age by menstrual dating. This results in many birth weight points at term appearing at later gestational ages than they actually should be, leading to an artifactual flattening of the growth curve at term and setting artificial low standards that result in an underestimation of growth restriction in this period. Most published standards have included only ultrasound-dated pregnancies [22-24, 2628].

The importance of using standards free from pathology has been stressed; otherwise, assessment of fetal growth is done against an inaccurate optimal weight. Therefore, exclusion of mothers with known risk factors for either low (hypertension, congenital anomalies, multiple pregnancies and preterm deliveries) or high birth weight (diabetes) is warranted. Controversy exists whether smokers should be excluded. While is seems logical to exclude these women to get standards reflecting optimal rather than real birth weight, most series $[22,24,28,45$, 47] have included smoking in the model since it is highly correlated with several of the other covariates [48]. Hence, its exclusion may skew the population towards a nonsmoker profile that may not be representative of the general population. Despite its inclusion to construct standards, it is agreed [49] that the smoking coefficient should not be used to prospectively adjust the optimal weight. Although including this negative coefficient would likely improve the prediction, not adjusting for this factor improves the identification of growth restriction due to smoking or other causes. Thus, the expected optimal weight is calculated as if each mother is a non-smoker.

\section{Analysis}

Although several standards have been published [22$28,50]$, methodological approaches to construct customized standards have remained very similar to the original report [45]. In short, optimal birth weight at term is modeled by linear regression, which takes into account the above-mentioned biological characteristics. The formula of Hadlock et al. [51], a model that predicts fetal weight for gestational age, has been the most widely used standard to individually derive optimal fetal weight for each gestational age. Other authors have used other formulae to extrapolate backwards the optimal weight with very similar results [22]. From that optimal fetal weight, the 
Fig. 2. Examples of customized charts using Gestation Related Optimal Weight software version 7.5.1 (www.gestation. net). The charts can be used to plot previous baby weights and ultrasound-estimated fetal weights in the current pregnancy (right $\mathrm{Y}$ axis) as well as fundal height measurements for serial assessment (left Y axis). The horizontal axis shows the day and month of the start of each week of gestation, calculated by the software on the basis of the estimated date of confinement. The 3 curves on the chart are the 50th centile and the 10th and 90th centile limits, representing the predicted range of optimal growth for each pregnancy, after adjustment for maternal height, weight, parity and ethnic origin. The pregnancy details are shown on the top left of the chart, with maternal height in $\mathrm{cm}$ and maternal weight in $\mathrm{kg}$. The example shows 2 mothers - 'Mrs. Small' and 'Mrs. Large' - with 2 different sets of characteristics. A previously born baby girl weighing 3,000 g at 40.0 weeks is illustrated as being of average size (49th birth weight centile) for Mrs. Small (a), but SGA (5th centile) for Mrs. Large (b). DOB = Date of birth; EDD $=\mathrm{ex}^{-}$ pected date of delivery.
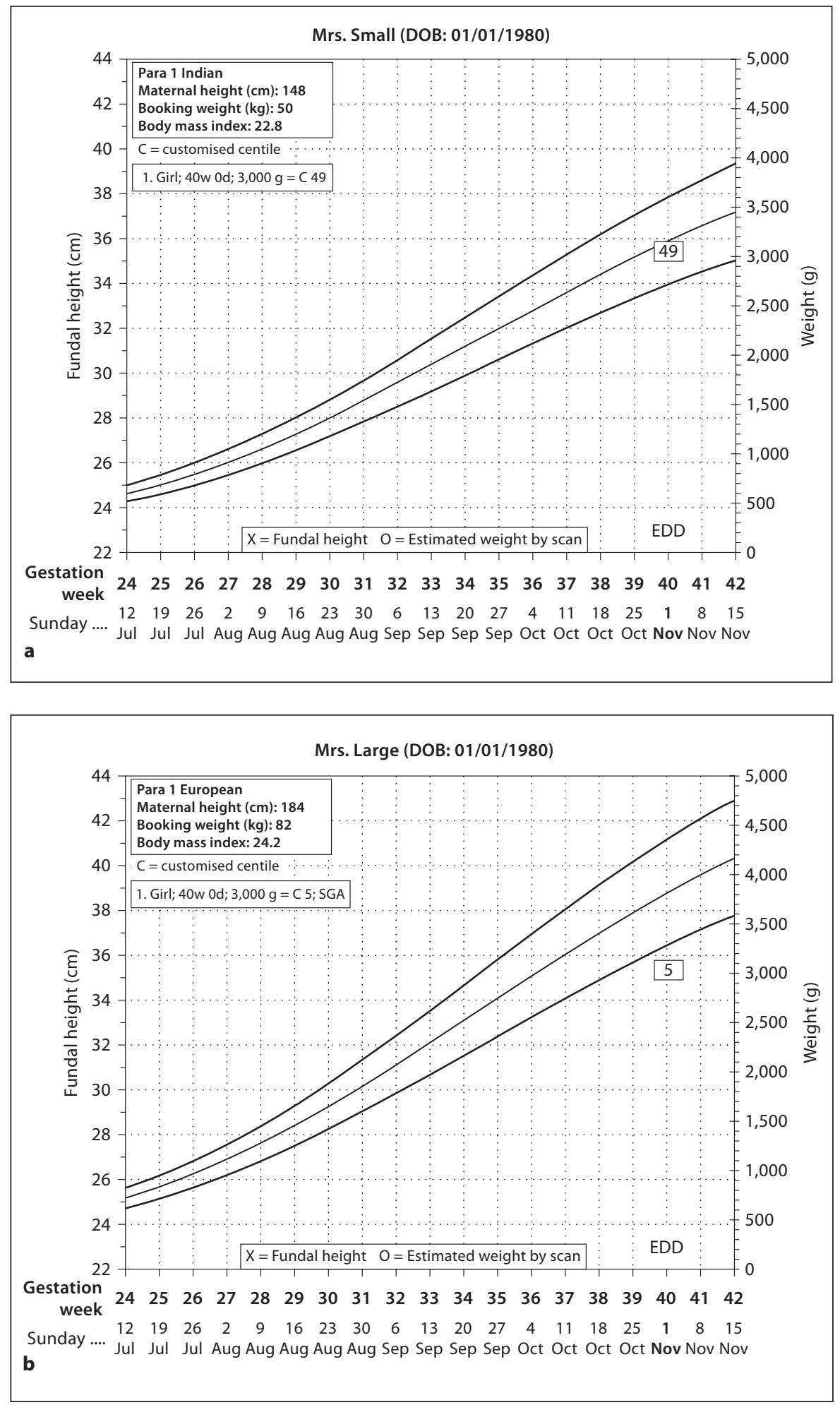
limits are calculated as a proportion of the standard variation at term, which assumes normal distribution and constant standard deviation throughout the third trimester.

Software for calculating customized fetal growth standards for different populations are freely available at www.gestation.net [52]. Figure 2 shows customized fetal growth charts for 2 different women, illustrating the concept of customization.

\section{Evidence for Customizing the Birth Weight Standard}

The use of customized birth weight standards has been demonstrated to be superior over population-based standards in the prediction of an abnormal 5-min Apgar score, hospital stay length, admission to the intensive care unit, hypoglycemia, need for neonatal resuscitation, neurological adverse outcome and perinatal death $[22,27,41$, $47,50,53,54]$. On the other hand, those neonates with a normal customized birth weight have been found to have a perinatal outcome comparable with the general population $[27,47,54,55]$, even in those SGA cases only according to population standards. The inference of these findings is that SGA according to customized standards and growth restriction are equivalent, and it has been claimed that customized SGA could be used as reliable proxy of growth restriction [49]. Figueras et al. [12] analyzed the relationship between umbilical artery Doppler and customized standards and concluded that normal antenatal umbilical artery Doppler cannot be taken as an indicator of low risk in pregnancies where the fetus is SGA according to customized standards. The adverse effects associated with customized SGA have also been demonstrated for long-term consequences such as cerebral palsy [56] and metabolic disturbances as impaired insulin secretion [57].

It has been suggested that the superiority of customized over population-based standards is mainly due to the former method classifying more preterm deliveries as SGA, which could account to a large extent for the adverse perinatal outcome $[41,58]$. However, this argument fails to explain the reported increased risk of stillbirth of customized SGA $[27,47,53]$. It has also been argued that the limitation of population standards in the prediction of adverse outcome in preterm neonates is overcome when fetal instead of neonatal standards are used [58]. However, in pregnancies with neonatal morbidity, customized standards identified $34 \%$ more pregnancies as abnormally grown than fetal growth standards [22], fur- ther supporting the concept that customized SGA babies are intrinsically at increased risk, regardless of gestational age. In a recent study of customized and uncustomized fetal weight standards, various maternal size and parity subgroups were examined, with the finding that SGA by the customized standard shows a consistently and significantly higher perinatal mortality risk than when SGA is defined by the population standard [59].

In conclusion, there is good quality evidence (grade II) to recommend adjusting birth weight for maternal and pregnancy variables to improve the distinction between constitutional and pathological 'smallness'.

\section{Evidence for Customizing Fetal Growth Charts}

Although it has been demonstrated that some maternal and fetal characteristics have an association with fetal ultrasound biometry $[29,60]$, there are no published customized standards derived from estimated fetal weight. However, the predicted 'term optimal (birth)weight' can be backwardly extrapolated into the intrauterine period, by combining it with a 'proportionality' curve derived from an in utero fetal weight standard [7]. Such antenatal growth curves have been found to reflect variation in population subgroups in low-risk [19] as well as high-risk [61] populations. By adjusting for individual variation, customized growth curves reduce false-positive diagnoses of fetal growth restriction [62] and are likely to reduce intervention: in a multi-ethnic population in the UK, 109 women were induced for suspected IUGR diagnosed on the basis of conventional fetal growth charts; retrospective application of customized charts found that the majority of cases (58\%) were in fact pregnancies where the babies' weight was within the normal weight range if assessed by customized charts [63]. In a Dutch study of 220 women with an elevated risk of placental insufficiency, serial ultrasound estimations of fetal weight plotted on customized standards resulted in a sensitivity for growth restriction of $83 \%$ for a falsepositive rate of $11 \%$ [55]. However, no comparison was made with uncustomized, population-based standards. Another uncontrolled study [64] found customized centiles only moderately useful (sensitivity $42 \%$, specificity $90 \%$ ) in the prediction of IUGR, using a lower-quartile neonatal ponderal index as outcome indicator. However, the investigators did not use the customized growth chart for serial assessment, but only calculated a centile from the last measurement. Thus, the accuracy of prediction was likely to have been a function of the accu- 
racy of ultrasound-estimated fetal weight. Further work is needed to assess the usefulness of customized fetal weight curves.

In many settings, fundus-symphysis height is used as a screening tool for fetal growth assessment. Fundal height measurement has been found to be subject to the same individual variation as birth weight and fetal weight [65]. A prospective nonrandomized controlled trial in the UK [66] found use of customized fundal height assessment improved the antenatal detection of fetal growth restriction (29-48\%) and, moreover, reduced the number of unnecessary referrals for ultrasound. The study was not powered to assess the effect on pregnancy outcome, but a larger study in Sweden has shown that increased antenatal detection of SGA per se does result in a lower risk of adverse outcome [67].

\section{Conclusions}

Maternal and fetal physiological characteristics have been described which account for a substantial part of the variation in birth weight at term. These characteristics can be used to calculate an individualized optimal weight. There is good quality evidence to recommend adjusting for maternal and pregnancy variables to improve the distinction between constitutional and pathological 'smallness' amongst fetuses and neonates. While observational studies also support antenatal application for the assessment of fetal growth, further prospective studies are needed to assess and quantify the effectiveness of customized versus conventional charts in reducing adverse pregnancy outcome.

\section{References}

$\checkmark 1$ Kady S, Gardosi J: Perinatal mortality and fetal growth restriction. Best Pract Res Clin Obstet Gynaecol 2004;18:397-410.

-2 Jarvis S, et al: Cerebral palsy and intrauterine growth in single births: European collaborative study. Lancet 2003;362:1106-1111.

-3 Leitner Y, et al: Neurodevelopmental outcome of children with intrauterine growth retardation: a longitudinal, 10-year prospective study. J Child Neurol 2007;22:580-587.

4 Godfrey KM, Barker DJ: Fetal nutrition and adult disease. Am J Clin Nutr 2000;71(suppl 5):1344S-1352S.

$\checkmark 5$ Lee PA, et al: International Small for Gestational Age Advisory Board consensus development conference statement: management of short children born small for gestational age, April 24-October 1, 2001. Pediatrics 2003;111:1253-1261.

-6 Lackman F, et al: Fetal umbilical cord oxygen values and birth to placental weight ratio in relation to size at birth. Am J Obstet Gynecol 2001;185:674-682.

7 Royal College of Obstetrics and Gynaecology Green-Top Guidelines: The Investigation and Management of the Small-for-Gestational-Age Fetus. London, RCOG, 2002.

8 SOGC Clinical Practice Guidelines. The use of fetal Doppler in obstetrics. J Obstet Gynecol Can 2003;25:601-607.

$\checkmark 9$ ACOG committee opinion. Utility of antepartum umbilical artery Doppler velocimetry in intrauterine growth restriction. Number 188, October 1997 (replaces No 116, November 1992). Committee on Obstetric Practice. American College of Obstetricians and Gynecologists. Int J Gynaecol Obstet 1997;59:269-270
10 Neilson JP, Alfirevic Z: Doppler ultrasound for fetal assessment in high risk pregnancies. Cochrane Database Syst Rev 2000: CD000073.

-11 McCowan LM, Harding JE, Stewart AW: Umbilical artery Doppler studies in small for gestational age babies reflect disease severity. BJOG 2000;107:916-925.

12 Figueras F, et al: Predictiveness of antenatal umbilical artery Doppler for adverse pregnancy outcome in small-for-gestational-age babies according to customised birthweight centiles: population-based study. BJOG 2008:115:590-594.

13 Valcamonico A, et al: Absent end-diastolic velocity in umbilical artery: risk of neonatal morbidity and brain damage. Am J Obstet Gynecol 1994;170:796-801.

-14 Soothill PW, et al: Prediction of morbidity in small and normally grown fetuses by fetal heart rate variability, biophysical profile score and umbilical artery Doppler studies. Br J Obstet Gynaecol 1993;100:742-745.

-15 Soothill PW, Bobrow CS, Holmes R: Small for gestational age is not a diagnosis. Ultrasound Obstet Gynecol 1999;13:225-228.

16 Bobrow CS, Soothill PW: Fetal growth velocity: a cautionary tale. Lancet 1999;353:1460.

- 17 Hershkovitz R, et al: Fetal cerebral blood flow redistribution in late gestation: identification of compromise in small fetuses with normal umbilical artery Doppler. Ultrasound Obstet Gynecol 2000;15:209-212.

18 Chang TC, et al: Prediction of perinatal morbidity at term in small fetuses: comparison of fetal growth and Doppler ultrasound. $\mathrm{Br} \mathrm{J}$ Obstet Gynaecol 1994;101:422-427.
19 Mongelli M, Gardosi J: Longitudinal study of fetal growth in subgroups of a low-risk population. Ultrasound Obstet Gynecol 1995;6: 340-344.

20 Wilcox M, et al: Birth weight from pregnancies dated by ultrasonography in a multicultural British population. BMJ 1993;307:588591.

21 Gardosi J, et al: An adjustable fetal weight standard. Ultrasound Obstet Gynecol 1995; 6:168-174.

22 Bukowski R, et al: Individualized norms of optimal fetal growth: fetal growth potential. Obstet Gynecol 2008;111:1065-1076.

23 Figueras F, et al: Customized birthweight standards for a Spanish population. Eur J Obstet Gynecol Reprod Biol 2008;136:2024.

24 McCowan L, et al: A customised birthweight centile calculator developed for a New Zealand population. Aust NZ J Obstet Gynaecol 2004;44:428-431.

25 Pain S, et al: Customised birthweight: coefficients for an Australian population and validation of the model. Aust NZ J Obstet Gynaecol 2006;46:388-394.

26 Sahota DS, et al: Customized birth weight: coefficients and validation of models in a UK population. Ultrasound Obstet Gynecol 2008;32:884-889.

27 Ego A, et al: Customized versus populationbased birth weight standards for identifying growth restricted infants: a French multicenter study. Am J Obstet Gynecol 2006;194: 1042-1049.

28 Mongelli M, et al: A customized birthweight centile calculator developed for an Australian population. Aust NZ J Obstet Gynaecol 2007;47:128-131. 
29 Drooger JC, et al: Ethnic differences in prenatal growth and the association with maternal and fetal characteristics. Ultrasound $\mathrm{Ob}-$ stet Gynecol 2005;26:115-122.

-30 Graafmans WC, et al: Birth weight and perinatal mortality: a comparison of 'optimal' birth weight in seven Western European countries. Epidemiology 2002;13:569-574.

- 31 Arntzen A, Nybo Andersen AM: Social determinants for infant mortality in the Nordic countries, 1980-2001. Scand J Public Health 2004;32:381-389.

-32 Arntzen A, et al: Socioeconomic status and risk of infant death. A population-based study of trends in Norway, 1967-1998. Int J Epidemiol 2004;33:279-288.

- 33 Raatikainen K, Heiskanen N, Heinonen S: Marriage still protects pregnancy. BJOG 2005;112:1411-1416.

34 Yip R: Altitude and birth weight. J Pediatr 1987;111:869-876.

35 Morrison J, et al: The influence of paternal height and weight on birth-weight. Aust NZ J Obstet Gynaecol 1991;31:114-116.

36 Windham GC, et al: Prenatal active or passive tobacco smoke exposure and the risk of preterm delivery or low birth weight. Epidemiology 2000;11:427-433.

37 Rasmussen S, Irgens LM: The effects of smoking and hypertensive disorders on fetal growth. BMC Pregnancy Childbirth 2006;6: 16.

38 Pollack H, Lantz PM, Frohna JG: Maternal smoking and adverse birth outcomes among singletons and twins. Am J Public Health 2000;90:395-400.

39 Gardosi J, Francis A: A customized standard to assess fetal growth in an American population. Am J Obstet Gynecol DOI: 10.1016/ j.ajog.2009.04.035.

-40 Nikkila A, Kallen B, Marsal K: Fetal growth and congenital malformations. Ultrasound Obstet Gynecol 2007;29:289-295.

-41 Groom KM, et al: Small-for-gestational-age infants classified by customized or population birthweight centiles: impact of gestational age at delivery. Am J Obstet Gynecol 2007;197:239 e1-e5.

-42 Gardosi JO: Prematurity and fetal growth restriction. Early Hum Dev 2005;81:43-49.
43 Zeitlin J, et al: The relationship between intrauterine growth restriction and preterm delivery: an empirical approach using data from a European case-control study. BJOG 2000;107:750-758.

44 Santamaria R, et al: Tablas españolas de pesos neonatales según edad gestacional. Barcelona, Laboratorios Menarini, 1998.

45 Gardosi J, et al: Customised antenatal growth charts. Lancet 1992;339:283-287.

46 Verburg BO, et al: New charts for ultrasound dating of pregnancy and assessment of fetal growth: longitudinal data from a population-based cohort study. Ultrasound Obstet Gynecol 2008;31:388-396.

47 Clausson B, et al: Perinatal outcome in SGA births defined by customised versus population-based birthweight standards. BJOG 2001;108:830-834.

48 Bonellie SR: Effect of maternal age, smoking and deprivation on birthweight. Paediatr Perinat Epidemiol 2001;15:19-26.

49 Gardosi J: Customized fetal growth standards: rationale and clinical application. Semin Perinatol 2004;28:33-40.

50 Sciscione AC, Gorman R, Callan NA: Adjustment of birth weight standards for maternal and infant characteristics improves the prediction of outcome in the small-forgestational-age infant. Am J Obstet Gynecol 1996;175:544-547.

51 Hadlock FP, Harrist RB, Martinez-Poyer J: In utero analysis of fetal growth: a sonographic weight standard. Radiology 1991; 181:129-133.

52 Gardosi J, et al: Gestation related optimal weight (GROW) program. 2003. www.gestation.net.

53 Figueras F, et al: Customized birthweight standards accurately predict perinatal morbidity. Arch Dis Child Fetal Neonatal Ed 2007;92:F277-F280.

54 McCowan LM, Harding JE, Stewart AW: Customized birthweight centiles predict SGA pregnancies with perinatal morbidity. BJOG 2005;112:1026-1033.

55 De Jong CL, et al: Customized fetal weight limits for antenatal detection of fetal growth restriction. Ultrasound Obstet Gynecol 2000;15:36-40.
56 Jacobsson B, et al: Cerebral palsy and restricted growth status at birth: populationbased case-control study. BJOG 2008;115: 1250-1255.

-57 Verkauskiene R, et al: Birth weight and longterm metabolic outcomes: does the definition of smallness matter? Horm Res 2008; 70: 309-315.

58 Zhang X, et al: The use of customised versus population-based birthweight standards in predicting perinatal mortality. BJOG 2007; 114:474-477.

59 Gardosi J, Clausson B, Francis A: The value of customized centiles in assessing perinatal mortality risk associated with parity and maternal size. BJOG DOI: 10.111/j.14710528.2009.02245.

60 Schwarzler P, et al: Sex-specific antenatal reference growth charts for uncomplicated singleton pregnancies at 15-40 weeks of gestation. Ultrasound Obstet Gynecol 2004;23: 23-29.

61 de Jong CL, et al: Fetal weight gain in a serially scanned high-risk population. Ultrasound Obstet Gynecol 1998;11:39-43.

62 Mongelli M, Gardosi J: Reduction of falsepositive diagnosis of fetal growth restriction by application of customized fetal growth standards. Obstet Gynecol 1996;88:844848.

63 Dua A, Schram C: An investigation into the applicability of customised charts for the assessment of fetal growth in antenatal population at Blackburn, Lancashire, UK. J Obstet Gynaecol 2006;26:411-413.

64 Owen P, et al: Prediction of intrauterine growth restriction with customised estimated fetal weight centiles. BJOG 2003;110:411415.

65 Mongelli M, Gardosi J: Symphysis-fundus height and pregnancy characteristics in ultrasound-dated pregnancies. Obstet Gynecol 1999;94:591-594.

66 Gardosi J, Francis A: Controlled trial of fundal height measurement plotted on customised antenatal growth charts. $\mathrm{Br} \mathrm{J}$ Obstet Gynaecol 1999;106:309-317.

67 Lindqvist PG, Molin J: Does antenatal identification of small-for-gestational age fetuses significantly improve their outcome? Ultrasound Obstet Gynecol 2005;25:258-264. 